\title{
Antibiotic exposure windows and the efficacy of immune checkpoint blockers in patients with cancer: a meta-analysis
}

\author{
Litang Huang ${ }^{1 \#}$, Xi Chen ${ }^{2 \#}$, Li Zhou ${ }^{3}$, Qiuli Xü ${ }^{3}$, Jingyuan Xie ${ }^{3}$, Ping Zhan ${ }^{3}$, Tangfeng Lv $^{3}$, Yong Song ${ }^{1,3} \wedge$ \\ ${ }^{1}$ Department of Respiratory and Critical Care Medicine, Affiliated Jinling Hospital, School of Medicine, Southeast University, Nanjing, China; \\ ${ }^{2}$ Department of Critical Care Medicine, Zhongda Hospital, School of Medicine, Southeast University, Nanjing, China; ${ }^{3}$ Department of Respiratory \\ and Critical Care Medicine, Affiliated Jinling Hospital, Medical School of Nanjing University, Nanjing, China \\ Contributions: (I) Conception and design: L Huang, X Chen; (II) Administrative support: Y Song; (III) Provision of study materials or patients: L \\ Huang, X Chen; (IV) Collection and assembly of data: L Zhou, Q Xu; (V) Data analysis and interpretation: J Xie, Ping Zhan; (VI) Manuscript \\ writing: All authors; (VII) Final approval of manuscript: All authors. \\ \#These authors contributed equally to this work. \\ Correspondence to: Tangfeng Lv, PhD. Department of Respiratory and Critical Care Medicine, Affiliated Jinling Hospital, Medical School \\ of Nanjing University, \#305, East Zhongshan Road, Nanjing 210002, China. Email: bairoushui@163.com; Yong Song, PhD. Department \\ of Respiratory and Critical Care Medicine, Affiliated Jinling Hospital, School of Medicine, Southeast University, Nanjing 210002, China. \\ Email: yong_song6310@yahoo.com.
}

Background Immune checkpoint blockers (ICBs) improve the survival of patients with cancer, but primary
or acquired drug resistance is inevitable. Intestinal microorganisms play an important role in immunotherapy
and antitumor response, and antibiotic use can cause changes in intestinal microbial abundance and
diversity. At present, the effects of antibiotic exposure on the anticancer activity of immunotherapy remain
controversial.

Methods: We performed a meta-analysis of relevant studies retrieved from electronic databases to assess the effects of the time window of antibiotic exposure on the efficacy of immune checkpoint inhibitors (ICIs). In accordance with the definition of antibiotic use in different articles, the time window of antibiotic exposure was divided into three groups, namely, Groups 1 (antibiotic use within 2 months before or after ICI), 2 (antibiotic use before ICI), and 3 (antibiotic use anytime during ICI).

Results: After retrieval from the PubMed and the Embase databases, 39 cohorts were included. In group 1, progression-free survival [PFS; hazard ratio $(\mathrm{HR})=1.81,95 \%$ confidence interval (CI): 1.40-2.34] and overall survival (OS; HR =1.81, 95\% CI: 1.43-2.28) were prolonged in patients without antibiotic use. In group 2, the subgroup analysis showed that antibiotic use had no effect on PFS (HR =0.90, 95\% CI: 0.65-1.26) and OS (HR $=1.53$, 95\% CI: 0.89-2.62) when the exposure window defined as 0-3 months. In Group 3, pooled results indicated that $\mathrm{PFS}$ ( $\mathrm{HR}=0.78,95 \% \mathrm{CI}: 0.65-0.93$ ) was prolonged in patients with antibiotic during immunotherapy, and no difference was observed in the OS data (HR =0.98, 95\% CI: 0.78-1.24) between the patients with antibiotic and without antibiotic.

Conclusions: Antibiotic use in shortly time (within before or after 2 months) around the initiation of immunotherapy was remarkably related to the efficacy of ICIs. A different scenario could be observed that during the long-term treatment of ICIs, the effect of antibiotic exposure seems to be eliminated.

Keywords: Antibiotics; gut microbiome; immune checkpoint inhibitors (ICIs); survival; meta-analysis

Submitted Oct 21, 2020. Accepted for publication Dec 04, 2020.

doi: 10.21037/apm-20-2076

View this article at: http://dx.doi.org/10.21037/apm-20-2076

^ ORCID: 0000-0003-4979-4131. 


\section{Introduction}

Immune checkpoint inhibitors (ICIs), including anticytotoxic T-lymphocyte-associated protein 4 (antiCTLA-4) and anti-programmed cell death protein-(L)-1 [anti-PD-(L)1] monoclonal antibodies (mAbs), reactivate the antitumor activity of $\mathrm{CD}^{+} \mathrm{T}$ cells by blocking $\mathrm{T}$ cell signals and are extensively approved in multiple cancers (1). In recent years, ICIs have dramatically revolutionized the management of multiple types of cancer. Patients with cancer have achieved overall response rates of $13.3-87 \%$, $18-23 \%$, and $11.9-19 \%$ by anti-PD-1, anti-PD-L1, and anti-CTLA-4 mAbs, respectively (2). However, some patients with advanced cancer have poor response to ICIs. In this regard, we seek to find the factors that influence the efficacy of ICIs for improved clinical drug use.

The gut microbiome has been demonstrated to affect cancer therapy especially the efficacy of checkpoint inhibitors in patients with advanced cancer (3). Routy et al. have found that the composition of the gut microbiome is different between the responders and the non-responders to ICIs and that fecal microbiota transplantation from responders can improve the efficacy of cancer immunotherapy in non-responders (4). Several retrospective studies have found that the poor efficacy of immunotherapy is associated with antibiotic (ATB) exposure, whereas Hogue et al. (5) have observed the opposite outcome. Also, some studies deny the association. Notably, these studies have not reached a consistent definition on the ATB use especially the time window of ATBs. Thus, we performed a meta-analysis to determine whether the use of ATBs before, during, or after immunotherapy affect the efficacy of ICIs in patients with cancer. This study aimed to explore many predictors for patient with ICIs. We present the following article in accordance with the PRISMA reporting checklist (available at http://dx.doi.org/10.21037/apm-20-2076).

\section{Methods}

\section{Literature search}

We conducted a systematic review in the PubMed and the Embase databases by using the terms "(immune checkpoint inhibitor [Title/Abstract]) OR immune checkpoint inhibitors [Title/Abstract]) OR immune checkpoint blockade [Title/Abstract]) OR ICI [Title/Abstract]) OR ICIs [Title/Abstract]) OR ICB [Title/Abstract]) OR immunotherapy [Title/Abstract]) OR immunotherapies [Title/Abstract]" and references from relevant articles in the latest 5 years up to Nov. 7, 2020. The included articles were subjected to a dual review, and the references of the included studies were manually reviewed for any additional publication. We searched the PROSPERO database without restricted and no articles were found. Our registration number was CRD42020155823. As we performed a metaanalysis about researches of published studies, no need application for ethics approval.

\section{Quality assessment and data extraction}

The data from each study that met the inclusion criteria were independently extracted by two authors (Litang Huang and Xi Chen). Any problem with data extraction was resolved by discussion. The retrieved and the extracted data included the author's name, year of publication, country, study design, cancer types, number of samples (number of patients exposed to ATBs), type of ICIs, ATB window, and outcomes [progression-free survival (PFS)/overall survival (OS), associated hazard ratio (HR), and $95 \%$ confidence interval (CI)]. If data were available in both sources, the source with more complete data were prioritized.

\section{Grouping}

Here, we divided the included studies into three groups in accordance with the time windows of exposure. Group 1 was administered with ATBs within 2 months before or after immunotherapy. Group 2 was injected with ATBs before immunotherapy. Group 3 was exposed to ATBs at any time during the immunotherapy (Figure 1).

\section{Statistical analysis}

The survival outcomes, including OS and PFS, were obtained. The effect of the time window of ATB exposure on the survival of patients with immunotherapy was determined using HRs and 95\% CIs. Furthermore, the association between ATB exposure window and ICI efficacy was included. A meta-analysis was performed to compute the weighted average of PFS or OS reported for patients with and without exposure to ATB. The $I^{2}$ statistic and the $\mathrm{P}$ value were used to examine heterogeneity across articles for each outcome. A P value $\leq 0.05$ was defined as significant heterogeneity. We conducted the subgroup analysis to examine studies in accordance with the type of group (ATB exposure window). The publication bias was assessed using the Begg's test and funnel plots, and significant publication 


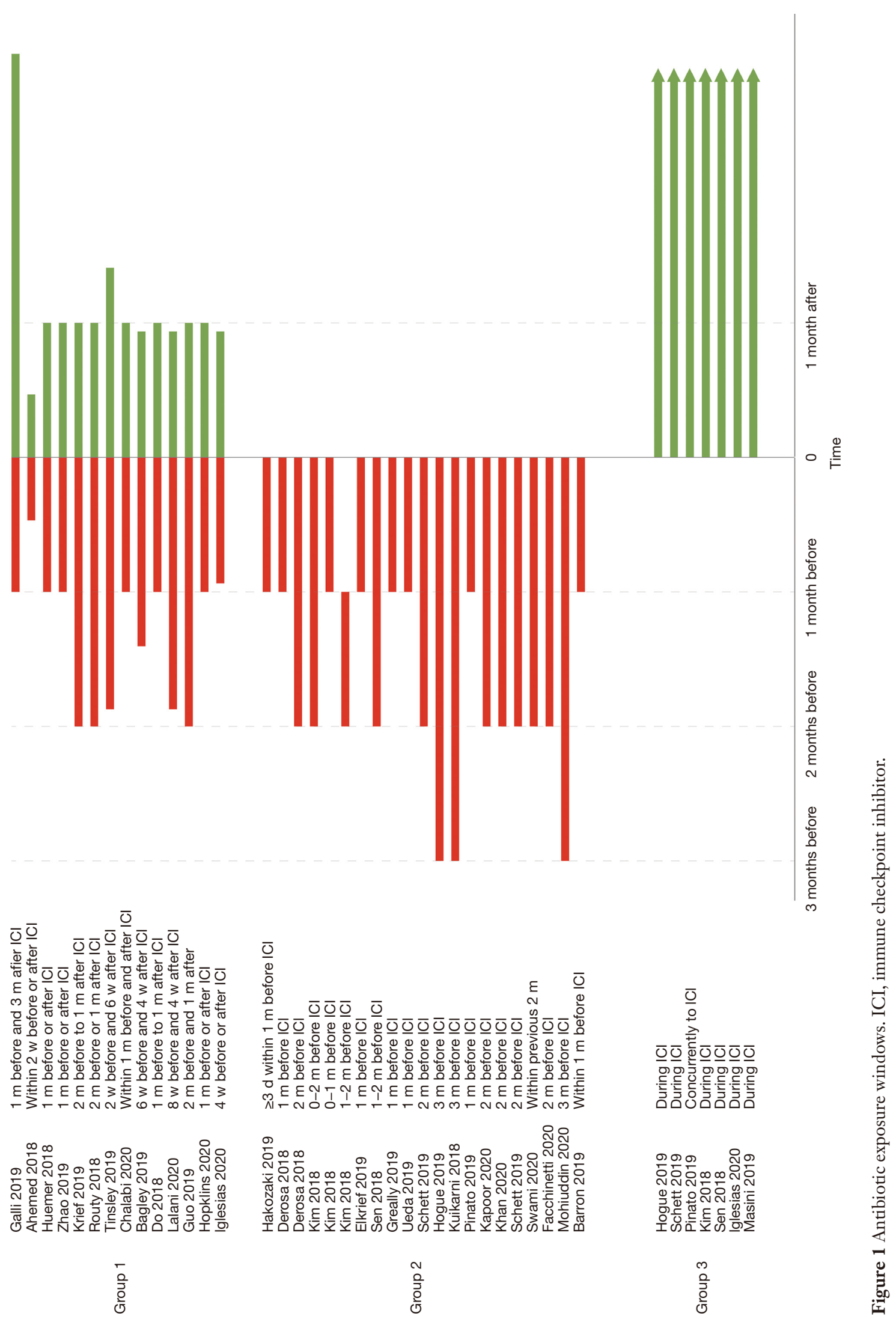




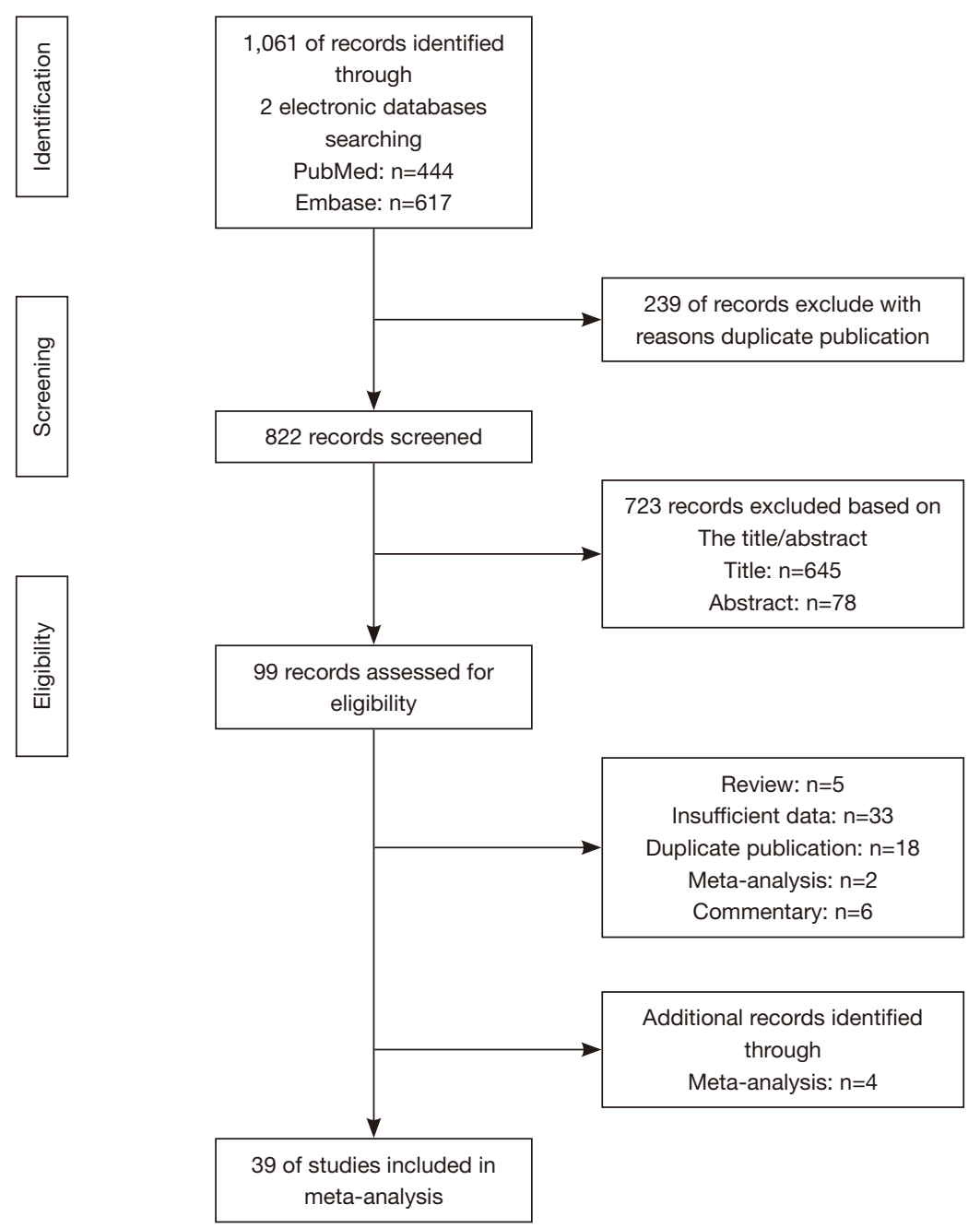

Figure 2 Literature search and study selection.

bias was defined as $\mathrm{P}<0.05$. All statistical analyses were conducted using the STATA version 15.

\section{Results}

A total of 1,061 relevant reports from the PubMed and the Embase databases were retrieved, and three more studies were identified. A total of 239 studies were removed after duplicate checking, and 723 studies were removed after reviewing the title or the abstract. After screening and eligibility assessment, 99 studies remained for fulltext screening. Sixty-four reports, including 5 reviews, 6 commentaries, 2 meta-analysis, 33 incomplete studies (lacking HR for PFS or OS), and 18 duplications, were subsequently excluded. Three records were identified through meta-analysis. Finally, 39 studies were included in our quantitative analysis (Figure 2). Twenty-eight studies were complete cohort studies, whereas the rest was shown only as abstract.

\section{Characteristics}

Table 1 shows the population distribution and the characteristics of the included studies. A total of 7,853 patients from 39 studies met our inclusion criteria. A total of 2,400 (30.6\%) patients were exposed to ATBs. The included studies were published between 2017 and 2020, and most studies were conducted in 2019 (48\%). Almost two-fifth $(37 \%)$ of the studies were from the United States. Of the 39 included studies, 2 and 35 were prospective and 
Table 1 Baseline characteristics

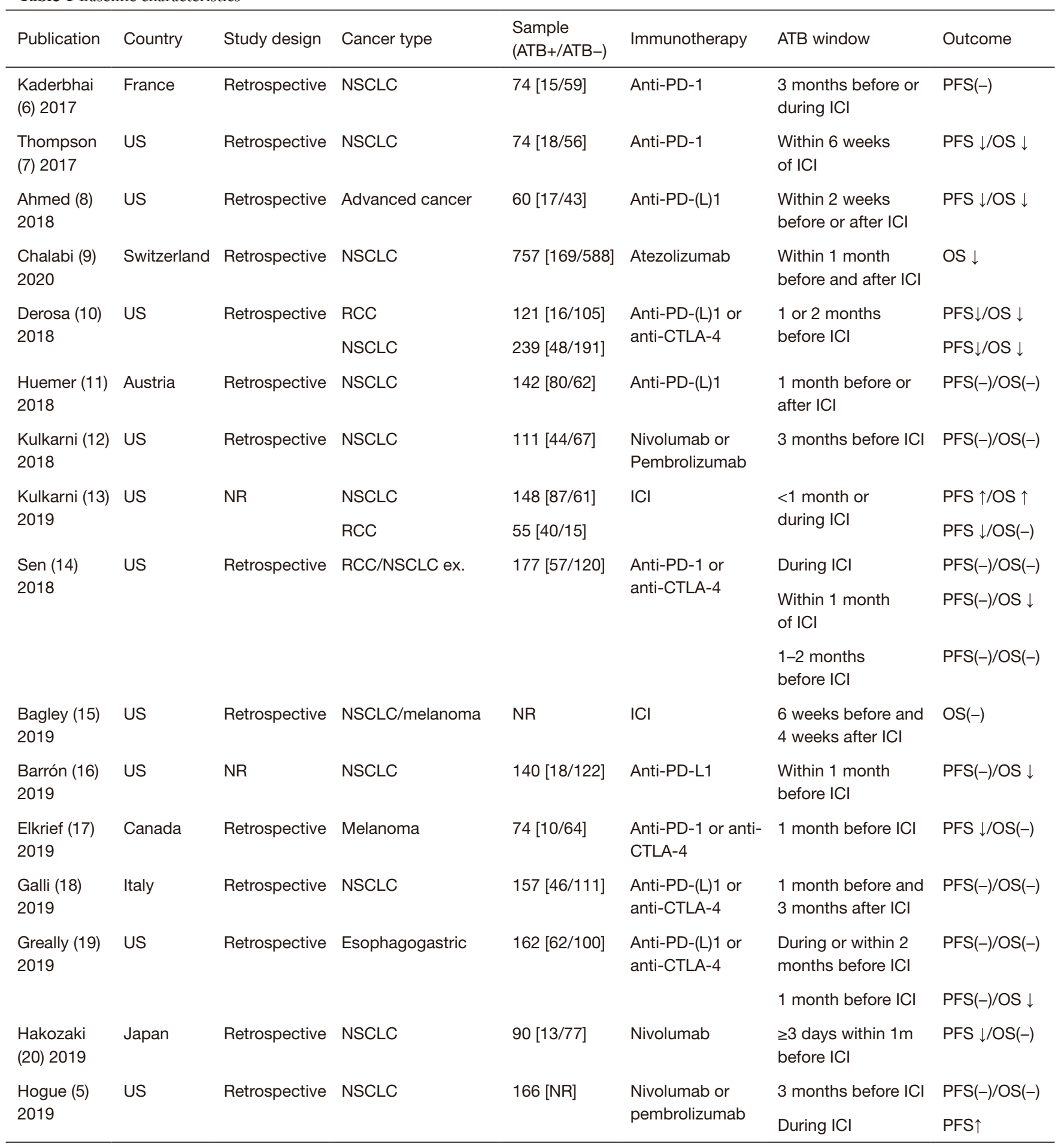

Table 1 (continued) 
Table 1 (continued)

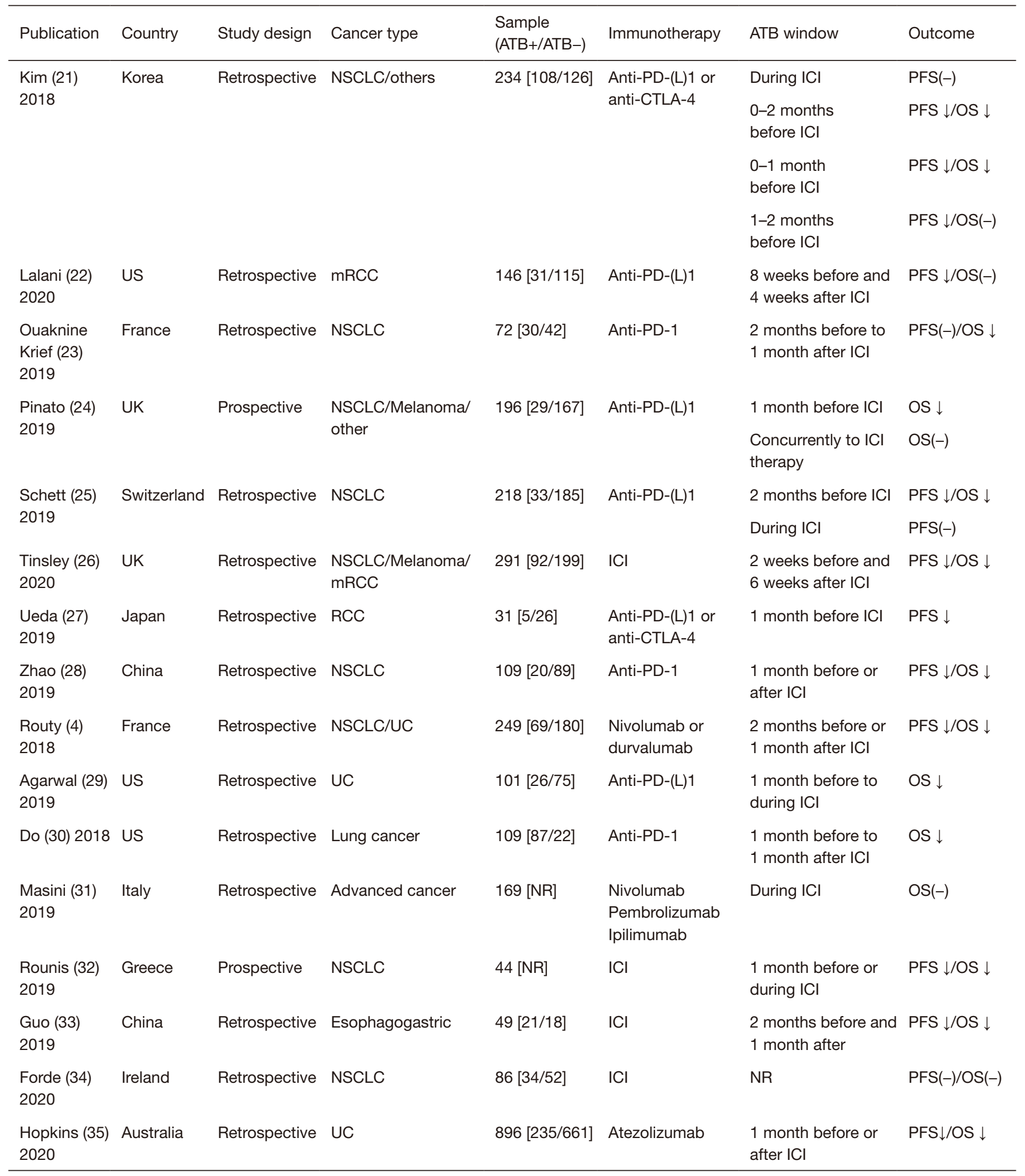

Table 1 (continued) 
Table 1 (continued)

\begin{tabular}{|c|c|c|c|c|c|c|c|}
\hline Publication & Country & Study design & Cancer type & $\begin{array}{l}\text { Sample } \\
\text { (ATB+/ATB-) }\end{array}$ & Immunotherapy & ATB window & Outcome \\
\hline \multirow[t]{2}{*}{$\begin{array}{l}\text { Iglesias- } \\
\text { Santamaría } \\
\text { (36) } 2020\end{array}$} & Spain & Retrospective & Advanced cancer & $102[60 / 42]$ & $\mathrm{ICl}$ & $\begin{array}{l}4 \text { weeks before or } \\
\text { after } \mathrm{ICl}\end{array}$ & PFS(-)/OS(-) \\
\hline & & & & [33/42] & & During $\mathrm{ICl}$ & PFS(-)/OS(-) \\
\hline \multirow{2}{*}{$\begin{array}{l}\text { Khan (38) } \\
2020\end{array}$} & NR & Retrospective & UC & $146[31 / 115]$ & $\mathrm{ICl}$ & 2 months before $\mathrm{ICl}$ & PFS(-)/OS(-) \\
\hline & & & & [44/46] & & 2 months after $\mathrm{ICl}$ & PFS(-)/OS(-) \\
\hline $\begin{array}{l}\text { Mohiuddin } \\
\text { (39) } 2020\end{array}$ & NR & Retrospective & Melanoma & $568[114 / 454]$ & $\mathrm{ICl}$ & 3 months before $\mathrm{ICl}$ & OS $\downarrow$ \\
\hline \multirow{3}{*}{$\begin{array}{l}\text { Abu-Sbeih } \\
\text { (41) } 2019\end{array}$} & US & Retrospective & Advanced cancer & $826[569 / 257]$ & $\mathrm{ICl}$ & After ICI & OS $\downarrow$ \\
\hline & & & & & & Before $\mathrm{ICl}$ & \\
\hline & & & & & & Before and after ICl & \\
\hline $\begin{array}{l}\text { Facchinetti } \\
\text { (42) } 2020\end{array}$ & Italy & Retrospective & SCLC & $143[36 / 107]$ & Pembrolizumab & 2 months before $\mathrm{ICl}$ & PFS(-)/OS(-) \\
\hline
\end{tabular}

ATB, antibiotic; NSCLC, non-small cell lung cancer; anti-PD-(L)1, anti-programmed cell death protein-(L)-1; PFS, progression-free survival; OS, overall survival; RCC, renal cell carcinoma; ICI, immune checkpoint inhibitor; anti-CTLA-4, anti-cytotoxic T-lymphocyte-associated protein 4; NR, not reported; US, United States; UC, urothelium carcinoma; UK, United Kingdom.

retrospective studies, and two studies did not mention the type of study. The patients were diagnosed with lung cancer (49\%), renal cell carcinoma (about 6\%), melanoma (about $13 \%)$ and other advanced cancers, including esophageal cancer and urothelium carcinoma. The ICIs included antiPD-(L)1 and anti-CTLA-4. The ATB window had different definitions in the studies (Table 1).

\section{Outcome data}

\section{Survival of group 1}

Group 1 included 3,237 patients from 14 studies. These patients mostly had non-small cell lung and urethral cancers. Pooled results showed that the ATB exposure were negatively associated with the PFS (HR $=1.81,95 \% \mathrm{CI}$ : $\left.1.40-2.34, I^{2}=55.0 \%\right)$ and the OS (HR $=1.81,95 \%$ CI: $1.43-$ $\left.2.28, I^{2}=61.5 \%\right)$ of patients who underwent immunotherapy (Figure 3). The PFS and the OS were analyzed using the random-effects models due to significant heterogeneity.

\section{Survival of group 2}

Group 2 was divided into three subgroups on the basis of the duration of the ATB exposure before immunotherapy. Subgroups 1, 2, and 3 were exposed to ATB before immunotherapy within 1,2 , and 3 months, respectively. The pooled results of subgroups 1 and 2 showed that ATB was a risk factor of poor OS (subgroup 1: $\mathrm{HR}=2.25,95 \%$ CI: $1.42-3.55$; subgroup 2: $\mathrm{HR}=1.57,95 \% \mathrm{CI}: 1.16-2.11$ ) and PFS (HR $=1.70,95 \% \mathrm{CI}: 1.35-2.14$; subgroup 2: $\mathrm{HR}=1.45,95 \% \mathrm{CI}: 1.04-2.02)$. However, the results of subgroup 3 showed that the ATB use was not related to the OS (HR $=1.53,95 \%$ CI: $0.89-2.62)$ and the PFS (HR $=0.90$, 95\% CI: $0.65-1.26)$ of patients with cancer who received immunotherapy. In subgroup 3, two cohorts for OS data and two cohorts for PFS data were available (Figure 4).

\section{Survival of group 3}

Four cohorts were included for analysis. Pooled results showed that ATB use could prolong the PFS $(\mathrm{HR}=0.78$, 
A

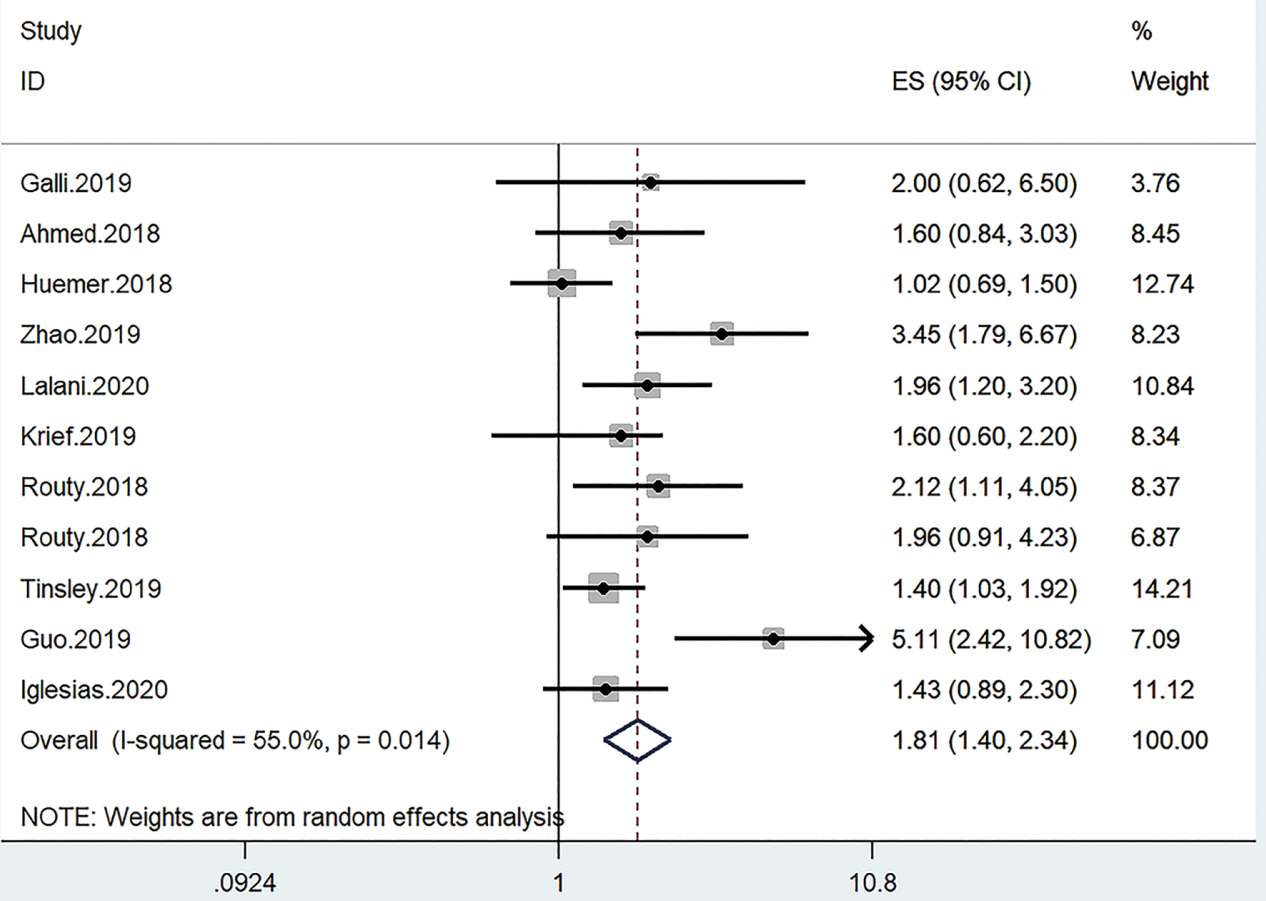

B

Study

ID
ES $(95 \% \mathrm{Cl}) \quad$ Weight

\section{Galli.2019}

Ahmed.2018

Huemer.2018

Zhao.2019

Lalani.2020

Chalabi.2018

Krief.2019

Routy.2018

Bagley.2019

Bagley.2019

Tinsley.2019

Do.2018

Guo.2019

Iglesias.2020

Abu-Sbeih.2019

Overall $(\mathrm{I}$-squared $=61.5 \%, p=0.001$ )

NOTE: Weights are from random effects analy'sis
$1.95(0.25,15.00) 1.17$

$2.90(1.10,8.10) 3.86$

$0.90(0.56,1.45) \quad 8.65$

$2.86(1.30,6.25) 5.31$

$1.44(0.75,2.77) \quad 6.54$

$1.32(1.06,1.63) \quad 12.17$

$2.20(1.10,4.80) 5.73$

$2.21(1.30,3.74) \quad 7.97$

$1.16(0.54,2.47) \quad 5.52$

$0.70(0.17,2.81) \quad 2.27$

$1.47(1.04,2.11) \quad 10.32$

$3.44(1.72,6.67) \quad 6.29$

$5.88(2.55,13.55) 4.92$

$1.53(0.88,2.66) \quad 7.66$

$2.21(1.70,2.86) \quad 11.61$

$1.81(1.43,2.28) \quad 100.00$

Figure 3 The associations between antibiotic exposure and PFS (A) and OS (B) in group 1. ES, effect size; CI, confidence interval; PFS, progression-free survival; OS, overall survival. 
A

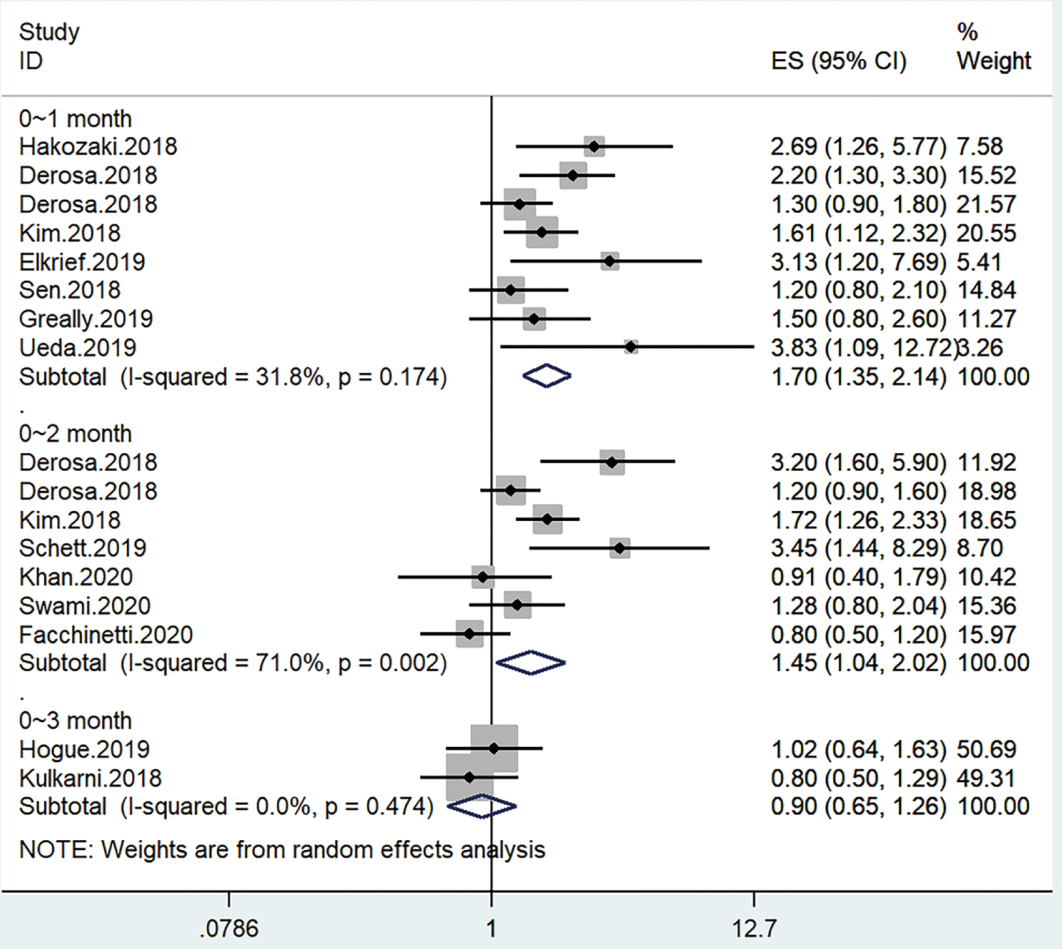

B

Study
ID

Figure 4 The associations between antibiotic exposure and PFS (A) and OS (B) in group 2. ES, effect size; CI, confidence interval; PFS, progression-free survival; OS, overall survival. 
95\% CI: 0.65-0.93) during immunotherapy. By contrast, the ATB use during immunotherapy was not related to the OS (HR $=0.98,95 \%$ CI: $0.78-1.24)$ of patients with cancer (Figure 5).

\section{Publication bias analysis}

The Begger's funnel plot was used to assess the publication bias in this meta-analysis. Results indicated no publication bias in any study, as evidenced by the symmetrical funnel plots (Figures S1-S3).

\section{Discussion}

Our meta-analysis has reported the relationship between the ATB exposure window and the efficacy of ICIs in patients with cancer. However, in the published metaanalysis, different results on the effect of ATBs on ICIs are observed. Huang et al. believe that ATB use was associated with poor survival in patients with immunotherapy (43). However, Wilson et al. have found that when a very broad definition of antibiotic exposure is adopted (antibiotic exposure anytime within the window 60 days before anytime after initiation of immunotherapy), the negative effect of antibiotic to PFS and OS was eliminated (44). Based on the work of Wilson $e t$ al., we have re-divided the included cohorts into three groups in accordance with the different definitions of the ATB exposure window to avoid the overlapping definitions of ATB time in different studies as much as possible. We have investigated the effects of ATB exposure on the antitumor efficacy and the survival of ICIs during immunotherapy. Group 1 (ATB use within 2 months before or after ICI) indicates that ATB use is a prognostic factor in immunotherapy. In group 2 (ATB use before ICI), the subgroup analysis shows that ATB use has no effect on immunotherapy when the exposure window is defined as 0-3 months. Although the ATB exposure window of the patients included cannot be completely distinguished, the cohorts have no detailed data about the patients exposed. The prolonged time between the exposure of ATB and the start of ICI may lead to the disappearance of adverse prognosis caused by ATB. Many studies suggest that ATBs may cause the poor efficacy of immunotherapy by affecting the abundance or imbalance of intestinal flora, and the gut flora may return to baseline after 42 days (44). In group 3 , pooled results show that ATB exposure is positively correlated with the PFS but not with the OS, Tinsley et al. noted that retrospective studies which failed to show any association between antibiotic therapy and ICI efficacy (26). Facchinetti et al. found that Eastern Cooperative Oncology Group performance status (ECOG PS) 2 was the only factor independently impacting on both PFS and OS (42), even though Hopkins et al. found the negative impact of antibiotic exposure, but the authors themselves are cautious in their interpretation of results, with a special situation was detected that ECOG PS was generally low in the cohort (35). Our results suggested that during the treatment of ICI, if ATB are required, perhaps, it may not cause the negative impact of efficacy of ICI. As we all known, patients with infection may cause bad PS, the findings about negative impact of antibiotic use which may be confounded by overall health status of patients that necessitates antibiotic use.

The limitations of our study are the same as those of several other published meta-analyses. The included studies are retrospective studies. Although we classify ATB exposure windows as best as we can, an overlap remains. In addition, the lack of baseline characteristics of the included patients, such as the type of ATB, specific infection site, duration of ATB use, and PS of patients, has prevented further subgroup analysis.

Several studies classify the patients who received ICIs into responders and non-responders in accordance with the best clinical response as assessed by the RECIST1.1 $(4,45)$. The baseline gut microbiome diversity and the relative abundance of the two groups are different, as shown by the higher relative abundance of the Akkermansia of the responder. The fecal microbiota of the two groups of patients are transplanted to specific pathogen-free mice. The mice transplanted with the microbiota of nonresponders had inferior response to ICI. Patients with high gut microbiome diversity and high relative abundance of some symbiotic bacteria are likely to benefit from ICIs. Studies have shown that the use of ATBs can affect the intestinal microbial diversity, thereby affecting the efficacy of ICIs. Different types of ATBs have different effects on the gut microbiome function. Mohiuddin et al. have found that the response of patients to ICIs is affected by the type of ATBs they use. Penicillin has the most serious adverse effects followed by cephalosporins and quinolones. However, vancomycin has no effect on the survival of patients (39). This article includes retrospective studies and cannot obtain the specific baseline characteristics of the included patients. Among all patients receiving immunotherapy, most patients using ATBs have respiratory or urinary tract infections. The immune characteristics, 
A

Study

$\%$

ID

ES $(95 \% \mathrm{Cl}) \quad$ Weight

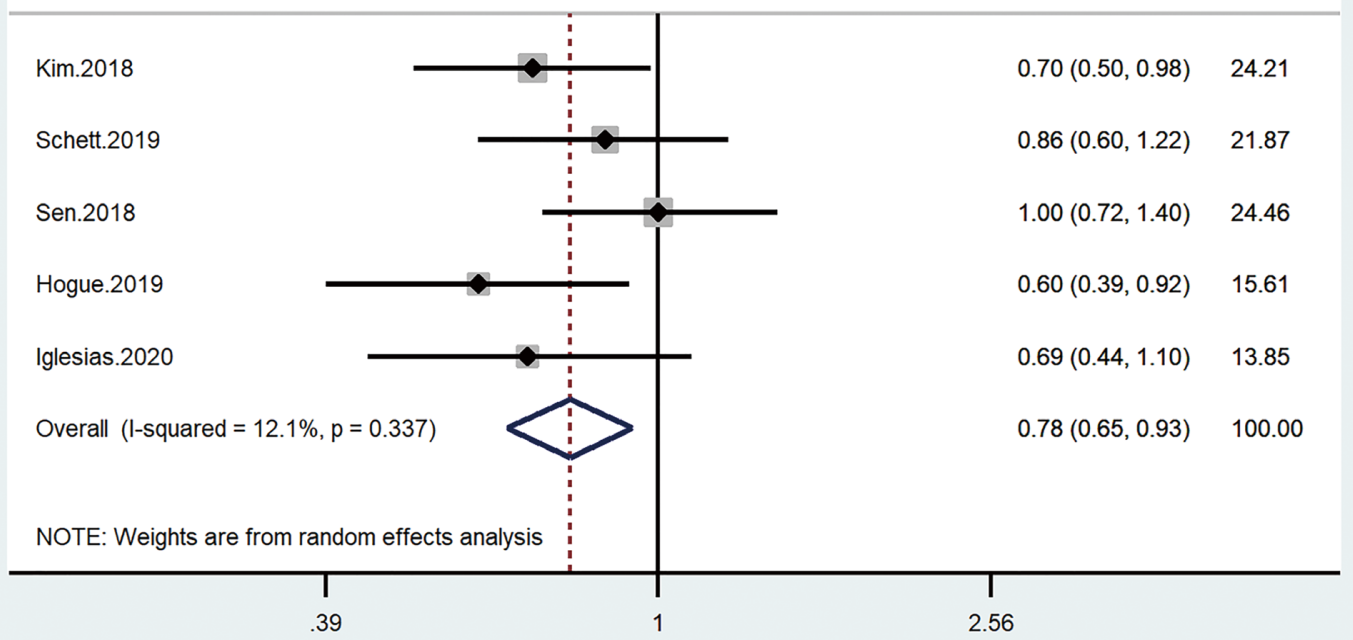

B

Study

ID
$\%$

ES $(95 \% \mathrm{Cl}) \quad$ Weight

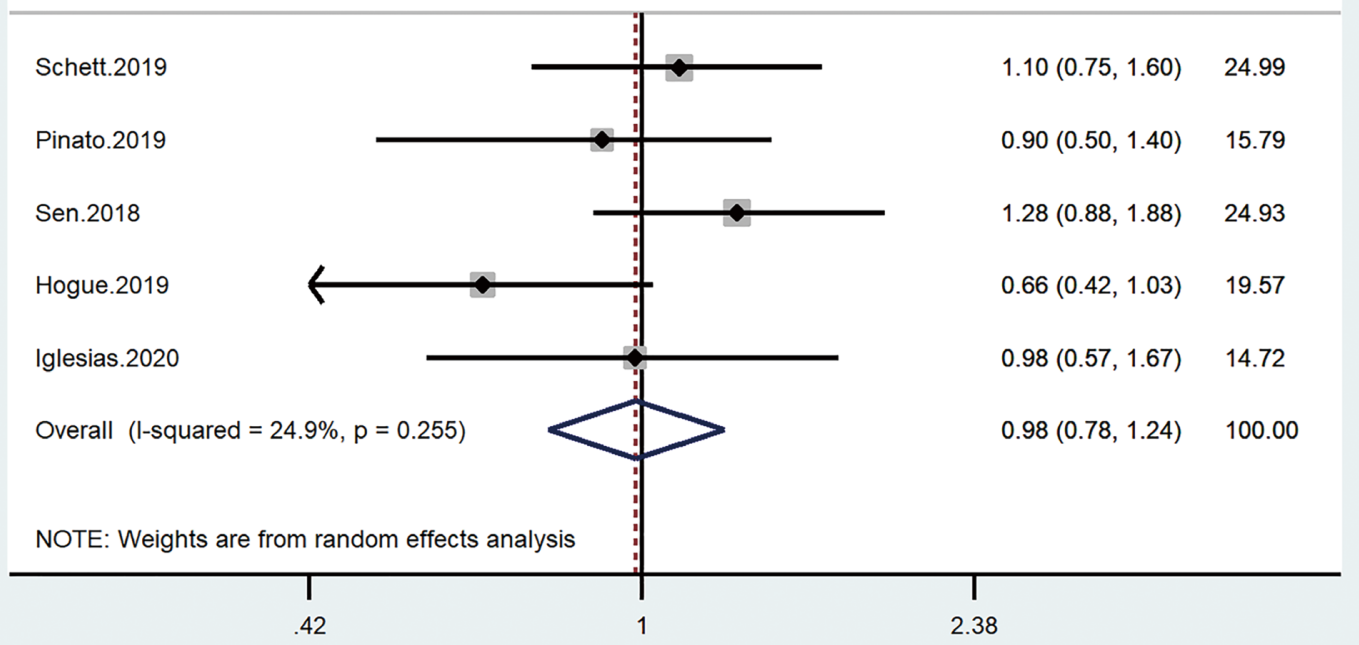

Figure 5 The associations between antibiotic exposure and PFS (A) and OS (B) in group 3. ES, effect size; CI, confidence interval; PFS, progression-free survival; OS, overall survival. 
baseline intestinal microbial, and ECOG status of patients with ATB exposure are different from those without ATB exposure. In some cases, such as patients with bacteremia, the use of ATBs is inevitable. ATBs improve the response of such patients to immunosuppressants by inhibiting pathogenic bacteria, and this finding may partly explain the high PFS of patients taking ATBs in group 3 (ATB use at any time during the ICIs). Therefore, we need to understand the baseline characteristics of patients using ATBs and the dynamic changes in their intestinal microbes after using different ATBs. Summarizing from the current research data, high-dose broad-spectrum ATBs (such as cephalosporins, $\beta$-lactams, and quinolones) may affect the intestinal flora, impair the efficacy of immunotherapy, and shorten the survival time of patients (reviewed and nonprospective data). The timing of ATBs is important. Before immunotherapy, if infections are present, the corresponding anti-infective treatment based on bacteriological evidence is recommended to be provided to avoid the prophylactic and the long-term use of ATBs.

\section{Conclusions}

This meta-analysis included 30 cohorts. Results showed that the survival of patients with cancer who underwent immunotherapy was associated with ATB exposure and that the timing of ATB use was an important factor. Different ATB exposure windows had different effects on the survival of patients with cancer. In the future, advanced prospective studies are needed to guide immunotherapy accurately and improve the patients' survival.

\section{Acknowledgments}

We are grateful to all the participants who have made this research possible.

Funding: This work was supported by the Jiangsu Provincial Social Development-Key Projects-Clinical Frontier Technologies (grant number BE2019719) and Jiangsu Provincial Social Development-General Program (grant number BE20197180).

\section{Footnote}

Reporting Checklist: The authors have completed the PRISMA reporting checklist. Available at http://dx.doi. org/10.21037/apm-20-2076
Peer Review File: Available at http://dx.doi.org/10.21037/ apm-20-2076

Conflicts of Interest: All authors have completed the ICMJE uniform disclosure form (available at http://dx.doi. org/10.21037/apm-20-2076). The authors have no conflicts of interest to declare.

Ethical Statement: The authors are accountable for all aspects of the work in ensuring that questions related to the accuracy or integrity of any part of the work are appropriately investigated and resolved.

Open Access Statement: This is an Open Access article distributed in accordance with the Creative Commons Attribution-NonCommercial-NoDerivs 4.0 International License (CC BY-NC-ND 4.0), which permits the noncommercial replication and distribution of the article with the strict proviso that no changes or edits are made and the original work is properly cited (including links to both the formal publication through the relevant DOI and the license). See: https://creativecommons.org/licenses/by-nc-nd/4.0/.

\section{References}

1. Ribas A, Wolchok JD. Cancer immunotherapy using checkpoint blockade. Science 2018;359:1350-5.

2. Seidel JA, Otsuka A, Kabashima K. Anti-PD-1 and AntiCTLA-4 Therapies in Cancer: Mechanisms of Action, Efficacy, and Limitations. Front Oncol 2018;8:86.

3. Gopalakrishnan V, Helmink BA, Spencer CN, et al. The Influence of the Gut Microbiome on Cancer, Immunity, and Cancer Immunotherapy. Cancer Cell 2018;33:570-80.

4. Routy B, Le Chatelier E, Derosa L, et al. Gut microbiome influences efficacy of PD-1-based immunotherapy against epithelial tumors. Science 2018;359:91-7.

5. Hogue C, Kuzel T, Borgia J, et al. P2.04-69 Impact of Antibiotic Usage on Survival During Checkpoint Inhibitor Treatment of Non-Small Cell Lung Cancer (NSCLC). J Thorac Oncol 2019;14:S735.

6. Kaderbhai C, Richard C, Fumet JD, et al. Antibiotic use does not appear to influence response to nivolumab. Anticancer Res 2017;37:3195-200.

7. Thompson J, Szabo A, Arce-Lara C, et al. P1.07008 Microbiome \& immunotherapy: Antibiotic use is associated with inferior survival for lung cancer patients receiving PD-1 inhibitors. J Thorac Oncol 2017;12:S1998. 
8. Ahmed J, Kumar A, Parikh K, et al. Use of broadspectrum antibiotics impacts outcome in patients treated with immune checkpoint inhibitors. Oncoimmunology 2018;7:e1507670.

9. Chalabi M, Cardona A, Nagarkar DR, et al. Efficacy of chemotherapy and atezolizumab in patients with nonsmall-cell lung cancer receiving antibiotics and proton pump inhibitors: pooled post hoc analyses of the OAK and POPLAR trials. Ann Oncol 2020;31:525-31.

10 Derosa L, Iebba V, Albiges L, et al. Gut microbiome composition to predict resistance in renal cell carcinoma (RCC) patients on nivolumab. J Clin Oncol 2018;36:abstr 4519.

11. Huemer F, Rinnerthaler G, Westphal T, et al. Impact of antibiotic treatment on immune-checkpoint blockade efficacy in advanced non-squamous non-small cell lung cancer. Oncotarget 2018;9:16512-20.

12. Kulkarni A, Patel M, Wang Y, et al. P573 Antibiotic use and clinical outcomes of PD-1 antagonists in advanced non-small cell lung cancers. 33rd Annual Meeting \& PreConference Programs of the Society for Immunotherapy of Cancer (SITC 2018). J Immunother Cancer 2018;6:115.

13. Kulkarni A, Kumar M, Pease DF, et al. Impact of antibiotics and proton pump inhibitors on clinical outcomes of immune check point blockers in advanced non-small cell lung cancers and metastatic renal cell cancer. J Clin Oncol 2019;37. doi: 10.1200/JCO.2019.37.15_ suppl.e20520.

14. Sen S, Carmagnani Pestana R, Hess K, et al. Impact of antibiotic use on survival in patients with advanced cancers treated on immune checkpoint inhibitor phase I clinical trials. Ann Oncol 2018;29:2396-8.

15. Bagley SJ, Dhopeshwarkar N, Narayan V, et al. Impact of antibiotics $(\mathrm{ABX})$ on overall survival (OS) in patients (pts) with advanced non-small-cell lung cancer (aNSCLC) and melanoma (aMel) treated with first-line immune checkpoint inhibition (ICI). J Clin Oncol 2019;37. doi: 10.1200/JCO.2019.37.15_suppl.e20643.

16. Barrón F, Cardona AF, Ruiz-Patiño A, et al. PD1.05 Relevance of Antibiotic Use on Clinical Activity of Immune Checkpoint Inhibitors in Hispanic Patients with Advanced Non-small-cell Lung Cancer (CLICAP-ABs). J Thorac Oncol 2019;14:S1168.

17. Elkrief A, Derosa L, Kroemer G, et al. The negative impact of antibiotics on outcomes in cancer patients treated with immunotherapy: a new independent prognostic factor? Ann Oncol 2019;30:1572-9.
18. Galli G, Triulzi T, Proto C, et al. Association between antibiotic-immunotherapy exposure ratio and outcome in metastatic non small cell lung cancer. Lung Cancer 2019;132:72-8.

19. Greally M, Chou JF, Chatila WK, et al. Clinical and Molecular Predictors of Response to Immune Checkpoint Inhibitors in Patients with Advanced Esophagogastric Cancer. Clin Cancer Res 2019;25:6160-9.

20. Hakozaki T, Okuma Y, Omori M, et al. Impact of prior antibiotic use on the efficacy of nivolumab for non-small cell lung cancer. Oncol Lett 2019;17:2946-52.

21. Kim H, Kim IH. Clinical association of antibiotics in immune checkpoint inhibitors for advanced cancer treatment. Ann Oncol 2018;29:ix170-2.

22. Lalani AA, Xie W, Braun DA, et al. Effect of Antibiotic Use on Outcomes with Systemic Therapies in Metastatic Renal Cell Carcinoma. Eur Urol Oncol 2020;3:372-81.

23. Ouaknine Krief J, Helly de Tauriers P, Dumenil C. et al. Role of antibiotic use, plasma citrulline and blood microbiome in advanced non-small cell lung cancer patients treated with nivolumab. J Immunother Cancer 2019;7:176.

24. Pinato DJ, Howlett S, Ottaviani D, et al. Association of Prior Antibiotic Treatment With Survival and Response to Immune Checkpoint Inhibitor Therapy in Patients With Cancer. JAMA Oncol 2019;5:1774-8. Erratum in: JAMA Oncol. 2020 Feb 1;6(2):302 . doi: 10.1001/ jamaoncol.2019.6921.

25. Schett A, Rothschild SI, Mauti LA, et al. Prognostic impact of the use of antibiotics in patients with advanced non-small cell lung cancer (NSCLC) receiving PD-(L)1 targeting monoclonal antibodies. Ann Oncol 2019;30:II58.

26. Tinsley N, Zhou C, Tan G, et al. Cumulative Antibiotic Use Significantly Decreases Efficacy of Checkpoint Inhibitors in Patients with Advanced Cancer. Oncologist 2020;25:55-63.

27. Ueda K, Yonekura S, Ogasawara N, et al. The impact of antibiotics on prognosis of metastatic renal cell carcinoma in Japanese patients treated with immune checkpoint inhibitors. Anticancer Res 2019;39:6265-71.

28. Zhao S, Gao G, Li W, et al. Antibiotics are associated with attenuated efficacy of anti-PD-1/PD-L1 therapies in Chinese patients with advanced non-small cell lung cancer. Lung Cancer 2019;130:10-7.

29. Agarwal A, Pond GR, Curran C, et al. Impact of concurrent medications on outcomes with PD1/PDL1 inhibitors for metastatic urothelial carcinoma. J Clin 
Oncol 2019;37:abstr 435.

30. Do TP, Hegde AM, Cherry CR, et al. Antibiotic use and overall survival in lung cancer patients receiving nivolumab. J Clin Oncol 2018;36. doi: 10.1200/ JCO.2018.36.15_suppl.e15109.

31. Masini C, Berselli A, Romagnani A, et al. Results of an Italian CORE-IMMUNO study: Safety and clinical-related biomarkers as predictors of immunotherapy (IT) benefit in real-world treatment of various advanced tumors (ATs). J Clin Oncol 2019;37. doi: 10.1200/JCO.2019.37.15_suppl. e14156.

32. Rounis K, Papadaki C, Makrakis D, et al. Correlation of various clinical, imaging and laboratory parameters with outcome in patients with metastatic non-small cell lung cancer (NSCLC) treated with immune checkpoint inhibitors (ICIs): Results from a prospective, observational, single institution study. Ann Oncol 2019;30:ii61.

33. Guo JC, Lin CC, Lin CY, et al. Neutrophil-to-lymphocyte Ratio and Use of Antibiotics Associated with Prognosis in Esophageal Squamous Cell Carcinoma Patients Receiving Immune Checkpoint Inhibitors. Anticancer Res 2019;39:5675-82.

34. Forde C, Gregory R, Scott JA, et al. Antibiotic use in advanced non-small cell lung cancer (NSCLC) patients receiving immune checkpoint inhibitors across the Northern Ireland Cancer Network. Lung Cancer 2020;139:S54.

35. Hopkins AM, Kichenadasse G, Karapetis CS, et al. Concomitant Antibiotic Use and Survival in Urothelial Carcinoma Treated with Atezolizumab. Eur Urol 2020;78:540-3.

36. Iglesias-Santamaría A. Impact of antibiotic use and other concomitant medications on the efficacy of immune checkpoint inhibitors in patients with advanced cancer. Clin Transl Oncol 2020;22:1481-90.

37. Kapoor A, Noronha V, Patil VM, et al. Concomitant use of antibiotics and immune checkpoint inhibitors in patients with solid neoplasms: Retrospective data from real-world settings. Ecancermedicalscience 2020;14:1038.

38. Khan MS, Radakovich N, Ornstein M, et al. Concomitant antibiotic use and its effect on immune-checkpoint inhibitor efficacy in patients with advanced urothelial carcinoma. Ann Oncol 2020;31:S597.

39. Mohiuddin JJ, Chu B, Facciabene A, et al. Association of antibiotic exposure with survival and toxicity in patients with melanoma receiving immunotherapy. J Natl Cancer Inst 2020; djaa057.

40. Swami U, Chennamadhavuni A, Borcherding N, et al. Multivariable Analysis of 169 Cases of Advanced Cutaneous Melanoma to Evaluate Antibiotic Exposure as Predictor of Survival to Anti-PD-1 Based Immunotherapies. Antibiotics (Basel) 2020;9:740.

41. Abu-Sbeih H, Herrera LN, Tang T, et al. Impact of antibiotic therapy on the development and response to treatment of immune checkpoint inhibitor-mediated diarrhea and colitis. J Immunother Cancer 2019;7:242.

42. Facchinetti F, Mazzaschi G, Barbieri F, et al. First-line pembrolizumab in advanced non-small cell lung cancer patients with poor performance status. Eur J Cancer 2020;130:155-67.

43. Huang XZ, Gao P, Song YX, et al. Antibiotic use and the efficacy of immune checkpoint inhibitors in cancer patients: a pooled analysis of 2740 cancer patients. Oncoimmunology 2019;8:e1665973.

44. Wilson BE, Routy B, Nagrial A, et al. The effect of antibiotics on clinical outcomes in immune-checkpoint blockade: a systematic review and meta-analysis of observational studies. Cancer Immunol Immunother 2020;69:343-54.

45. Jin Y, Dong H, Xia L, et al. The Diversity of Gut Microbiome is Associated With Favorable Responses to Anti-Programmed Death 1 Immunotherapy in Chinese Patients With NSCLC. J Thorac Oncol 2019;14:1378-89.
Cite this article as: Huang L, Chen X, Zhou L, Xu Q, Xie J, Zhan P, Lv T, Song Y. Antibiotic exposure windows and the efficacy of immune checkpoint blockers in patients with cancer: a meta-analysis. Ann Palliat Med 2021;10(3):2709-2722. doi: 10.21037/apm-20-2076 


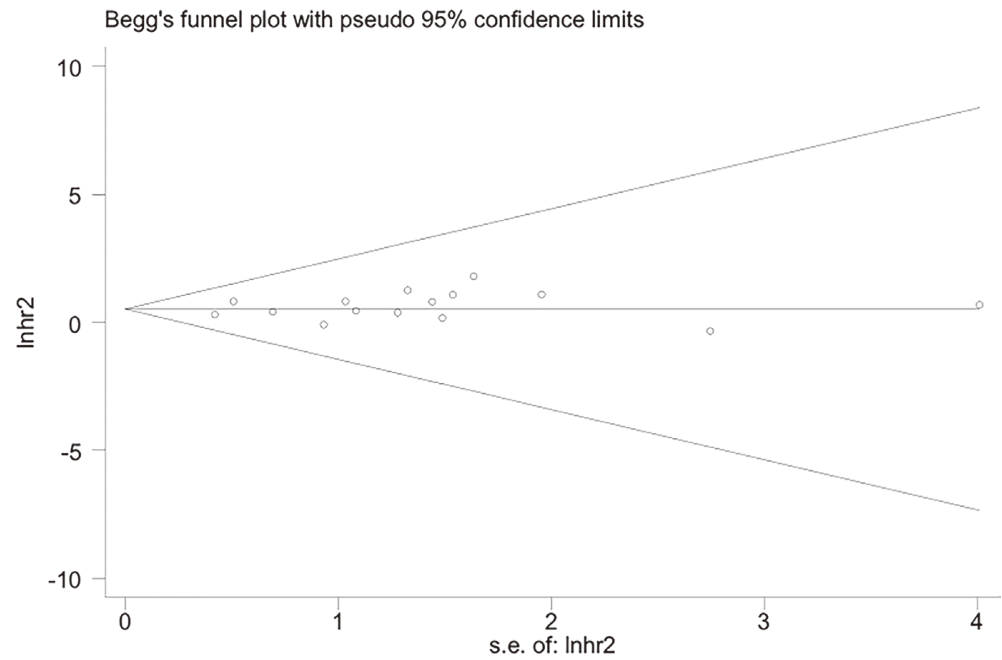

Figure S1 Funnel plot of overall survival in group 1.

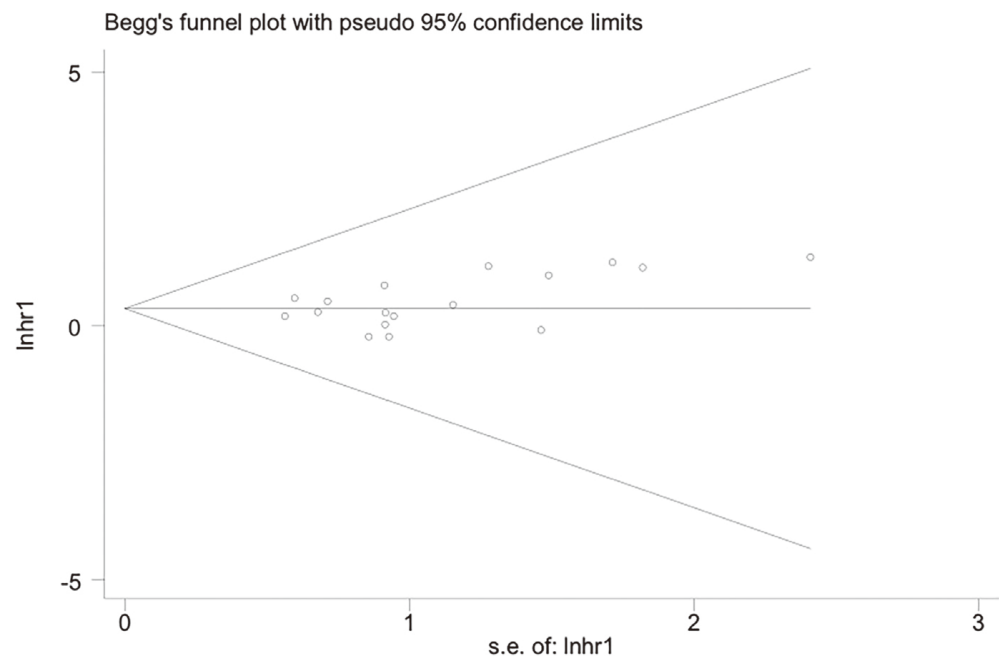

Figure S2 Funnel plot of progression-free survival in group 2. 


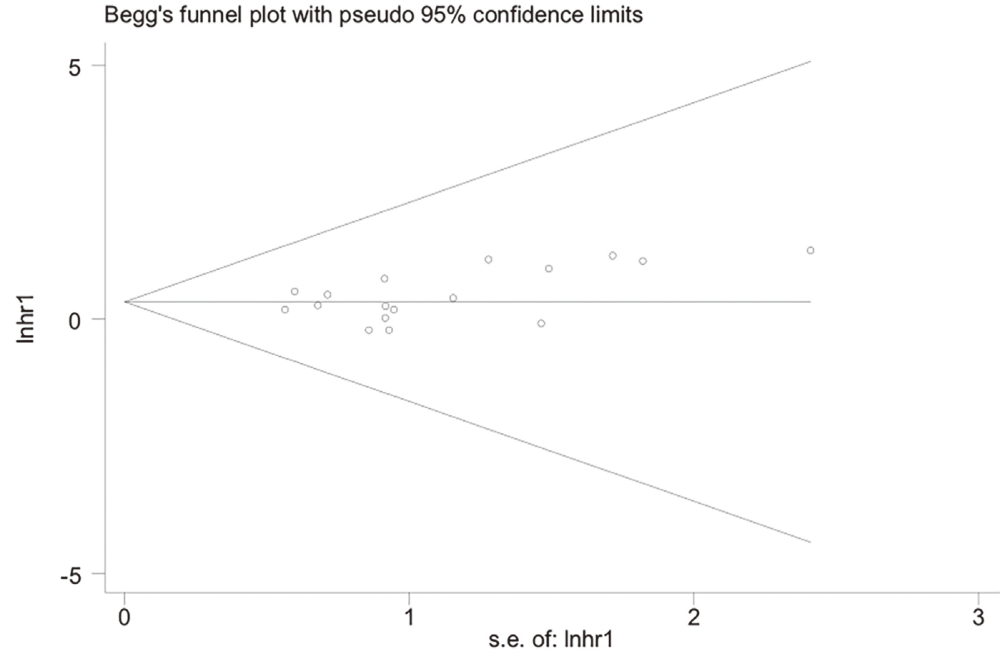

Figure S3 Funnel plot of overall survival in group 2. 